\title{
DOĞRUSAL HAREKETLİ SÜREKLİ MIKNATISLI SENKRON MOTORUN YAPAY SİNİR AĞ TABANLI SKALER HIZ DENETİMİ
}

\author{
Özcan OTKUN ${ }^{1}$, Ramazan Özgür DOĞAN ${ }^{1}$, Adem Sefa AKPINAR ${ }^{2}$ \\ ${ }^{1}$ Gümüşhane Üniversitesi, MYO, Bilgisayar Teknolojileri Bölümü, 29100, Gümüşhane \\ ${ }^{2}$ Karadeniz Teknik Üniversitesi, Mühendislik Fak., Elektrik-Elektronik Müh. Bölümü, 61100, Trabzon \\ ozcanotkun@gumushane.edu.tr, ramazan.dogan@msn.com, akpinar@ktu.edu.tr
}

(Geliş/Received: 14.10.2014; Kabul/Accepted: 14.07.2015)

ÖZET

\begin{abstract}
Günümüzde Yapay Sinir Ağları (YSA), farklı uygulamalarda, doğrusal olmayan birçok problemin çözümünde kullanılmaya başlanmıştır. Bu çalışmada, Doğrusal Hareketli Sürekli Mıknatıslı Senkron Motorun (DHSMSM) YSA tabanlı skaler (V/f) hız denetimi deneysel olarak gerçekleştirilmiştir. Skaler denetim için ürün modeli LGSV022IG5A-4 olan değişken frekanslı sürücü kullanılmıştır. Bu sürücünün frekansı C\# programlama diliyle hazırlanan YSA ile denetlenerek motorun hız denetimi gerçekleştirilmiştir. DHSMSM, farklı hız ve yük koşullarında çalıştırılarak elde edilen deneysel sonuçlar sunulmuş ve motorun hız denetimindeki başarım analizi yapılmıştır.
\end{abstract}

Anahtar kelimeler: Doğrusal hareketli motor, DHSMSM, YSA, skaler denetim, hız denetim

\section{NEURAL NETWORK BASED SCALAR SPEED CONTROL OF LINEAR PERMANENT MAGNET SYNCHRONOUS MOTOR}

\begin{abstract}
Nowadays, Artificial Neural Networks (ANN), in different applications, have been used in solving many problems of non-linear. In this study, ANN based Scalar (V / f) speed control of Linear Permanent Magnet Synchronous Motor (LPMSM) was carried out experimentally. For scalar control, variable frequency drive that is product of model LG- SV022IG5A-4 are used. This drive is controlled by the frequency of the C \# programming language prepared by the ANN and the motor speed control was performed. Obtained experimental results by running LPMSM at different speed and load conditions were presented and analysis of success in motor speed control was performed.
\end{abstract}

Keywords: Linear motor, LPMSM, YSA, scalar control, speed control

\section{GÍRISŞ (INTRODUCTION)}

Teknolojideki gelişmelere bağlı olarak doğrusal hareket önemini giderek arttırmaktadır. Doğrusal hareketi elde etmenin en kolay yolu elektrik motorlarıdır ve endüstriyel uygulamalarda sıkça kullanılmaktadır [1], [2]. Çevremize baktığımızda doğrusal hareketi elde etmek için döner hareket yapan motorların kullanıldığı çok sayıda örneği görebiliriz. Ancak, bu motorlarda döner hareketi doğrusal harekete dönüştürmek için kayış, dişli vb. gibi ek donanımlara ihtiyaç vardır. Bu durum sistem verimini olumsuz yönde etkilemektedir [2], [3]. Bu nedenle doğrusal hareketi ara düzeneklere ihtiyaç duymadan sağlayan Doğrusal Hareketli Motorlar (DHM) son yıllarda yaygın olarak kullanılmaya başlanmışıtır [3] [5].

Günümüzde döner hareketli motorların bütün türlerine benzer (Doğru Akım, Asenkron, Senkron, Relüktans vb.) DHM'ler bulunabilmektedir. $\mathrm{Bu}$ anlamda DHM'leri çok sayıda gruba ayırmak mümkündür [3]. Geçmişte doğru akım motorları uygulamada sıklıkla kullanılmasına rağmen güç elektroniği ve mikroişlemci teknolojisindeki gelişmelerle birlikte artık birçok uygulamada Doğrusal Hareketli Senkron 
ve Asenkron Motorların kullanılmaktadır [1], [5] [10]. Burada endüstriyel uygulamalar incelendiğinde; yüksek hızlanma kabiliyeti [11], yüksek itme kuvveti [8], yüksek motor gücüne sahip [12], aynı zamanda hassas pozisyonlama yapılabilen [6], [13], Doğrusal Hareketli Sürekli Miknatıslı Senkron Motorların (DHSMSM) tercih edildiği görülmektedir.

DHSMSM'ler yapı olarak değilse bile çalışma prensibi olarak döner hareketli motorlarla birbirine benzemektedir. $\mathrm{Bu}$ nedenle üç fazlı motorlar için kullanılan denetim yöntemleri DHM'ler içinde kullanılabilir [14]. Bu motorlar endüstride farklı farklı amaçlar için kullanıldıklarından, denetimleri içinde amaca uygun farklı denetim yöntemlerinin kullanıldığ̣ görülmektedir [1], [15]. Denetim yöntemleri genel olarak Skaler, Vektörel, PID ve Akıllı denetim yöntemleri olarak sınıflandırılabilir. Burada adı geçen denetim yöntemleri evirici devreler ve denetleyici devreler ile beraber kullanılmaktadır [16].

$\mathrm{Bu}$ motorların hız denetimlerinde basit yapılı olmaları nedeniyle geleneksel denetleyiciler (Skaler, Vektör, PID gibi.) sıklıkla kullanılmaktadır. Geleneksel denetleyicilerden skaler denetim yöntemi, düşük maliyetleri ve kolay uygulanabilirliği bakımından tercih edilmektedir [17], [18]. Ancak yapılan çalışmalara bakıldığında çok düşük hızlarda geleneksel denetleyicilerin istenilen denetim başarımını göstermediği ve başarımı arttırmak için çeşitli çalışmaların önerildiği görülmektedir [16], [19] - [24].

Geleneksel denetleyiciler endüstriyel alanda çok fazla kullanılmasına rağmen, sistemde modellenemeyen parametrelerden (elektrik motorlarının parametreleri) ötürü istenilen performansı gösteremezler [25]. Yani bu motorların sarg1 dirençleri sıcaklığa, sarg1 indüktansları manyetik doyuma bağlıdır. $\mathrm{Bu}$ durum denetim sisteminin dinamik davranışının bozulmasına ve kararsızlığa yol açmaktadır [26] - [29]. Bu nedenle akıllı denetim yöntemleri (Yapay Sinir Ağı, Bulanık Mantık, vb.) geliştirilmiştir. Yapılan çalışmalara bakıldığında, değişen sistem parametrelerine çabuk uyum sağlayan, basit ve anlaşılır olan, öğrenme ve genelleme yeteneğine sahip, doğrusal olmayan problemlerin çözümünde doğru sonuçlar üretebilen Yapay Sinir Ağlarının (YSA) tercih edildiği görülmektedir [30] - [34].

Literatürdeki hız denetim çalışmalarında, yalnız geleneksel denetleyiciler yerine, YSA veya YSA tabanlı geleneksel denetleyicilerin birlikte kullanıldı ̆̆ çalışmaların önerildiği görülmektedir [33], [35] - [38]. $\mathrm{Bu}$ anlamda DHSMSM'lerin denetimi üzerine yapılacak her türlü çalışmanın literatüre ve uygulamaya katkı yapacağı düşünülmektedir. Bu çalışmada, YSA tabanlı skaler denetim yöntemi kullanılarak, DHSMSM'nin hız denetimi deneysel olarak gerçekleştirilmiştir. Farklı hız (düşük hızlar) ve yük koşullarında motorun hız denetimi analiz edilmiştir.

\section{DHSMSM MODELİ (MODEL OF PMLSM)}

\subsection{DHSMSM'nin Matematiksel Modeli (Mathematical Models of the LPMSM)}

Üç fazlı alternatif akım motorlarının geçici ve kararlı durumdaki matematiksel ifadeleri hesaplama kolaylığı açısından uzay vektörleri ile tanımlanmaktadır. Ayrica bu motorların denetiminde yüksek performanslı sürücü geliştirmek için faz düzlemleri arasında dönüşüm gerçekleştirilir. Faz dönüşümleri kullanılarak motorun dinamik eşitliklerinin çözümü daha hızlı ve kolay olmaktadır [16]. Faz dönüşüm işlemleri;

$$
\begin{aligned}
& \text { - } \quad A B C \rightarrow \text { Clark } \rightarrow \alpha \beta \rightarrow \text { Park } \rightarrow d q \\
& \text { - } \quad \text { TersPark } \rightarrow \alpha \beta \rightarrow \text { TersClark } \rightarrow A B C,
\end{aligned}
$$

şeklinde gerçekleştirilir.

$\mathrm{Bu}$ duruma göre $A B C \rightarrow d q$ eksen sisteminin matematiksel ifadesi denklem 1 de görülmektedir.

$$
\left.\left[\begin{array}{l}
S_{d} \\
S_{q}
\end{array}\right]=\frac{2}{3}\left[\begin{array}{cc}
\cos (\theta) & \cos \left(\theta-\frac{2 \pi}{3}\right) \cos \left(\theta+\frac{2 \pi}{3}\right) \\
\sin (\theta) & \sin \left(\theta-\frac{2 \pi}{3}\right)
\end{array}\right\} \sin \left(\theta+\frac{2 \pi}{3}\right)\right]\left[\begin{array}{c}
S_{A} \\
S_{B} \\
S_{C}
\end{array}\right]
$$

Aynı şekilde $d q \rightarrow A B C \quad$ eksen sisteminin matematiksel ifadesi de denklem 2 de gösterilmektedir.

$$
\left[\begin{array}{c}
S_{A} \\
S_{B} \\
S_{C}
\end{array}\right]=\left[\begin{array}{cc}
\cos (\theta) & \sin (\theta) \\
\cos \left(\theta-\frac{2 \pi}{3}\right) & \sin \left(\theta-\frac{2 \pi}{3}\right) \\
\cos \left(\theta+\frac{2 \pi}{3}\right) & \sin \left(\theta+\frac{2 \pi}{3}\right)
\end{array}\right]\left[\begin{array}{c}
S_{d} \\
S_{q}
\end{array}\right]
$$

Burada $S$ vektörel olarak birincil yan (sargiların bulunduğu hareketli parça) akım, gerilim ve manyetik akı'yı ifade etmektedir.

DHSMSM'lerin çalışma prensibi döner hareketli motorlarla benzer kabul edilmektedir. $\mathrm{Bu}$ anlamda motorun yaygın olarak kullanılan iki eksen takımlı ( $d$ q) matematiksel modeli aşağıda verilmiştir [23], [25].

$$
\frac{d i_{d}}{d t}=-\frac{R}{L_{d}} i_{d}+w \frac{L_{q}}{L_{d}} i_{q}+\frac{1}{L_{d}} u_{d}
$$




$$
\frac{d i_{q}}{d t}=-\frac{R}{L_{q}} i_{q}-w \frac{L_{d}}{L_{q}} i_{d}-w \frac{\lambda_{s m}}{L_{q}}+\frac{1}{L_{q}} u_{q}
$$

Denklem 3 ve 4 de, $u_{d}, u_{q}$ d ve q eksenlerindeki gerilimler, $i_{d}, i_{q} \mathrm{~d}$ ve $\mathrm{q}$ eksenlerindeki akımlar, $R$ birincil yan sargi direnci, $L_{d}, L_{q}$ d ve q eksenlerindeki birincil yan sargı indüktansları, $w$ açısal hız ve $\lambda_{s m}$ sürekli mıknatıs akı bağıntısıdır.

Döner hareketteki açısal hız, doğrusal harekette doğrusal hıza (v) dönüşmektedir. Açısal hız;

$w=2 \pi f=v \frac{\pi}{\tau}$

Burada $\tau$ kutup adımı, $f$ çalışma frekansını göstermektedir. Burada doğrusal hız;

$v=2 f \tau$

DHSMSM nin elektromanyetik itme kuvveti;

$$
F_{i t m e}=\frac{3}{2} p \frac{\pi}{\tau}\left[\lambda_{S M}+\left(L_{d}-L_{q}\right) i_{d}\right] i_{q}
$$

SMDHSM'nin mekanik itme denklemi ise;

$$
F_{i t m e}=F_{h}+B v+M \frac{d v}{d t}
$$

Burada, $F_{h}$ harici (dış) kuvvet, $B$ sürtünme kuvveti, $M$ motorun ağırlığıdır.

\subsection{DHSMSM'nin Yapısı (Structure of LPMSM)}

Deneysel çalışma için tasarımı gerçekleştirilen motorun üst ve yandan görünümü Şekil 1'de gösterilmiştir [39].
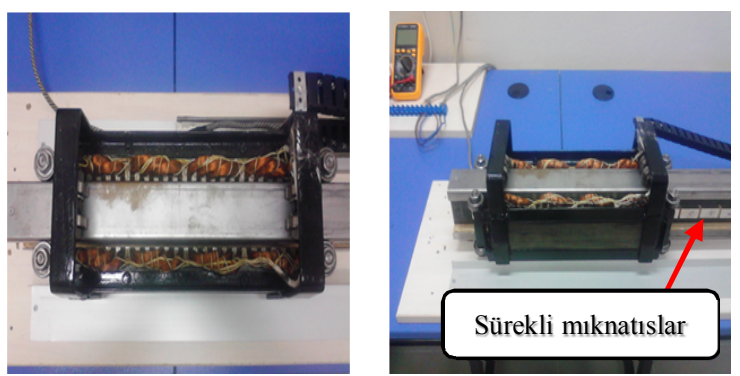

Şekil 1. DHSMSM yapısı (Structure of LPMSM)

Motora ait sürekli mıknatıs ölçüleri Tablo 1'de, motor parametreleri ise Tablo 2'de verilmiştir.
Tablo 1. Sürekli mıknatıs ölçüleri (Permanent magnet sizes)

\begin{tabular}{ll}
\hline \hline Parametreler & Değer \\
\hline Sürekli mıknatıs boyu & $40 \mathrm{~mm}$ \\
Sürekli mıknatıs eni & $20 \mathrm{~mm}$ \\
Sürekli mıknatıs kalınlığı & $10 \mathrm{~mm}$ \\
\hline \hline
\end{tabular}

Tablo 2. DHSMSM parametreleri (Parameters of LPMSM)

\begin{tabular}{clc}
\hline \hline Simge & \multicolumn{1}{c}{ Parametreler } & Değer \\
\hline$p$ & Kutup sayısı & 8 \\
$R$ & Sargı direnci & $11.58 \Omega$ \\
$\lambda_{S M}$ & Sürekli mıknatıs akısı & $0.485 \mathrm{~Wb}$ \\
$L_{d}$ & d ekseni indüktası & $29.5 \mathrm{mH}$ \\
$L_{q}$ & q ekseni indüktası & $29.5 \mathrm{mH}$ \\
$P$ & Motorun gücü & $0.1 \mathrm{~kW}$ \\
$\tau$ & Kutup adımı & $0.042 \mathrm{~m}$ \\
$M$ & Motor ağırlığı & $15.5 \mathrm{~kg}$ \\
\hline \hline
\end{tabular}

Deneye ait çalışma alanı Şekil 2'de gösterilmiştir.

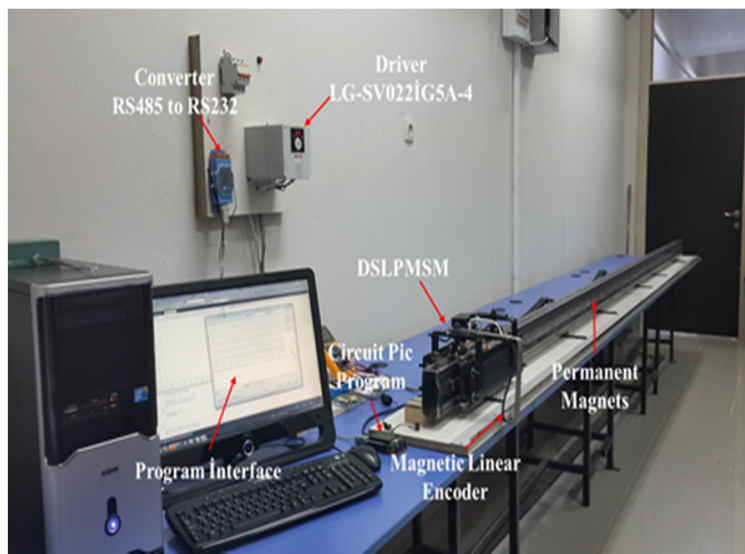

Şekil 2. Çalışma alanı (Study area)

\subsection{Kullanılan Sürücü (Used Drive)}

Motor denetimi için ürün kodu LG-SV022İG5A-4 sürücü kullanılmıştır. Kullanılan bu sürücü skaler kontrol (V/f) ve sensörsüz vektör kontrol yapabilmektedir. Minimum $0 \mathrm{~Hz}$ 'den maksimum 400 Hz'e kadar frekans çıkışı sağlayabilmektedir. Sürücü haberleşmesi seri port üzerinden yapılmaktadır. Dışarıdan programlanabilir giriş çıkış terminaline ve dâhili RS485 seri porta sahiptir. Bilgisayar veri girişi RS232 olduğundan RS485 to RS232 konvertör kullanılmıştır.

\section{YSA MODELİ (MODEL OF ANN)}

$\mathrm{Bu}$ çalışma da, doğrusal olmayan problemlerin çözümünde başarılı sonuçlar üreten Çok Katmanlı İleri Beslemeli Sinir Ağı (Multilayer Feedforward Network) kullanılmıştır. Ağın eğitiminde, danışmanlı öğrenme methodu ve hata geri yayılım algoritması (Error Back-Propagation Training) tercih edilmiştir. 


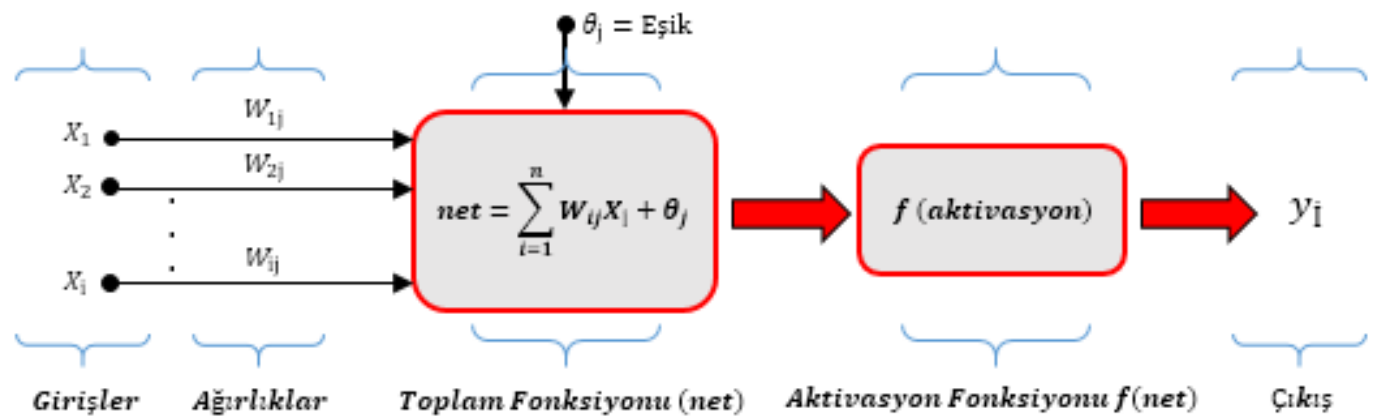

Şekil 3. YSA nın blok modeli (Block model of ANN)

Danışmanlı öğrenme de, sistem aldığı bilgilerle kıyaslama yapıp sinıflandırarak kendi kendine öğrenir. Geri yayılımlı öğrenme kuralı da, ağ çıkışındaki mevcut hata düzeyine göre her bir tabakadaki ağırlıkları yeniden hesaplamak için kullanılmaktadır.

Bir geri yayılımlı ağ modelinde giriş, gizli ve çıkış olmak üzere 3 katman bulunmakla birlikte, problemin özelliklerine göre gizli katman sayısını artırabilmek mümkündür. $\mathrm{Bu}$ duruma ait YSA'nın blok modeli Şekil 3'de gösterilmektedir [31], [39].

Öğrenme sırasında, giriş örnekleri belli bir sıra ile ağa verilir. Her bir çalışma örneği katmanlar boyunca sıra ile ilerleyerek çıkış örneği hesaplanana kadar ileri yayılır. Çıkışta elde edilen değer beklenen değer ile karşılaştırılıp fark yani hata bulunur. Hatalar, her katmanda geri besleme bağlantılarından 'girişler' olarak tekrar kullanılır.

Geriye doğru bağlantılar sadece 'öğrenme' için kullanılır. İleri doğru olan bağlantılar ise hem öğrenme hem de işlemsel amaçlı kullanılır [40].

\section{YSA TABANLI SKALER HIZ DENETIMI (ANN BASED SCALAR SPEED CONTROL)}

Bu çalışmada, YSA ile motor sürücüsünün frekansı değiştirilerek DHSMSM'nin istenen hıza ulaşması sağlanmıştır. $\mathrm{Bu}$ çalışmayla ilgili yapılan testler motorun yüklü ve yüksüz durumları için gerçekleştirilmiştir. Elde edilen sonuçlar grafiklerle gösterilmiştir. Sistemin çalışma aşamaları aşağıdaki gibi izah edilebilir:

1. Aşama: Bilgisayarda hazırlanan programın arayüzüne motorun gitmesi istenen hiz ve bu yolu gitmesi istenen zaman bilgileri girilir. Sisteme zaman girilmesinin nedeni motorun alabileceği yolun sınırlı olmasıdır $(480 \mathrm{~cm})$. Örneğin $50 \mathrm{~cm} / \mathrm{sn}$ için maksimum hareket süresi 9 sn olmalıdır.

2. Așama: YSA ya girilen hız değerine karşılık olması gereken frekans değeri 6 numaralı denklem yardımıyla hesaplanır. Aynı zamanda program çalışırken motor sürücüsünden anlık frekans değeri alınarak, YSA ya verilir. YSA, hesaplanan hızın olması gerekenden düşük olması durumunda frekansı azaltacak değilse frekansı arttıracaktır. Örnek eğitim seti Şekil 4'de gösterilmiştir.

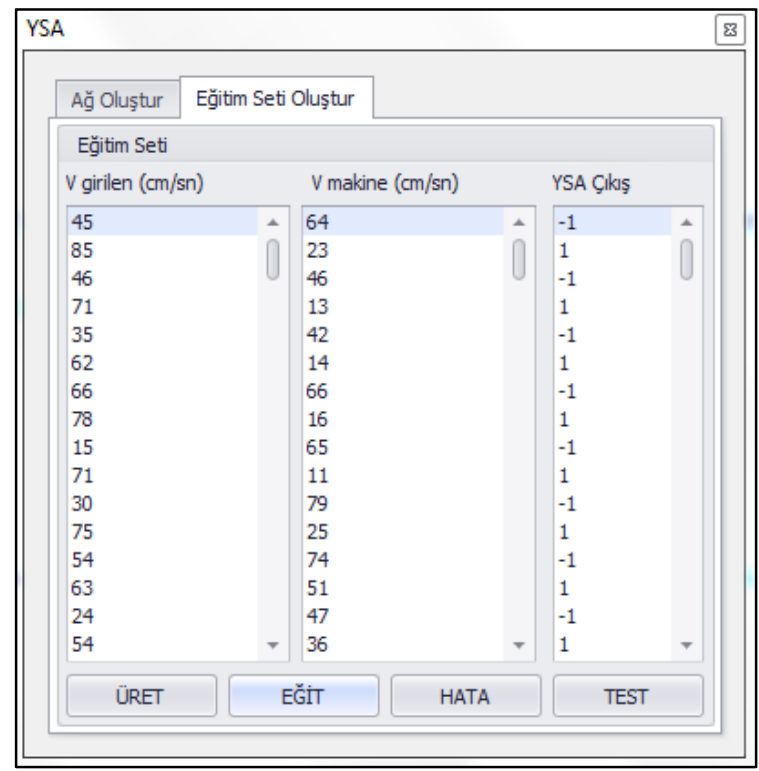

Şekil 4. Örnek YSA eğitim seti (Example ANN training set)

YSA değişebilen $v$ değerleri için rasgele değerler üretilerek bu değerlere karşılık gelecek $f$ değerlerini hesaplar. Burada hesaplanan her bir değere $f_{h}$ ve doğrusal hareketli motorun anlık frekansına $f_{t}$ dersek YSA blok yapısı Şekil 5'deki gibi kurulmuş olacaktır. Burada YSA'nın ürettiği çıtı $\Delta f$ değeridir ve bu değer doğrusal hareketli motorun bir sonraki frekansı $f_{t+1}$ in hesaplanmasında değişim miktarı olarak kullanılmaktadır. $f_{t+1}$ Denklem 9'da gösterilmiştir.

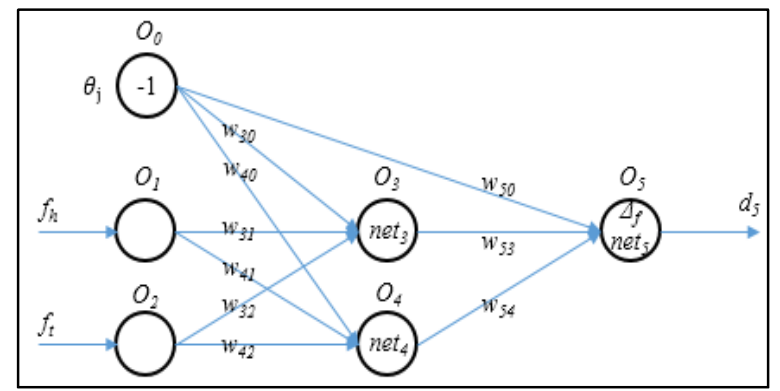

Şekil 5. Deneysel çalışmanın YSA yapısı (ANN structure of the experimental study) 


$$
f_{t+1}=f_{t}+\Delta f
$$

Burada, $\Delta f$ değeri anlık artış ya da azalış miktarı olarak kullanılacağı için YSA da kullanılan aktivasyon fonksiyonu +1 ile -1 aralığında değerler üretilecek şekilde gerçeklemelidir. $f_{h}$ YSA programı arayüzüne girilen hız değerine göre atanmaktadır.

Bulunan değer sürücüdeki değerden küçük ise YSA çıkışı "+1" yani frekansı arttır demektir. Eğer büyük ise YSA çıkışı "-1" yani frekansı azalt demektir. Bu işlem her 0.1 saniyede tekrarlanır. Zaman sıfır olduğunda ise motor durur.

Aktivasyon fonksiyonu olarak +1 ve -1 aralığında değer üreten bipolar sigmoid fonksiyonu denklemi kullanılmıştır [39].

$$
f(x)=\frac{2}{1+e^{x}}-1
$$

YSA da $\Delta f$ değeri $f_{t+1}$ anındaki değişim miktarını ifade edeceği için +1 ve -1 aralığında çıkış üreten bipolar sigmoid fonksiyonu:

$$
f(\text { net })=\frac{2}{1+e^{-n e t}}-1
$$

$\mathrm{Bu}$ fonksiyonun türevi;

$$
f^{\prime}(n e t)=\frac{1}{2}\left(1-n e t^{2}\right)
$$

YSA'nın bir adım ileri yönde hesaplanması aşağıdaki şekilde yapılmaktadır.

$$
\begin{aligned}
& \text { net }_{3}=O_{0} \cdot w_{30}+O_{1} \cdot w_{31}+O_{2} \cdot w_{32} \\
& \Rightarrow O_{3}=f\left(n e t_{3}\right)=\frac{1}{2} \cdot\left(1-n e t_{3}^{2}\right) \\
& \text { net }_{4}=O_{0} \cdot w_{40}+O_{1} \cdot w_{41}+O_{2} \cdot w_{42} \\
& \Rightarrow O_{4}=f\left(n e t_{4}\right)=\frac{1}{2} \cdot\left(1-n e t_{4}^{2}\right) \\
& n e t_{5}=O_{0} \cdot w_{50}+O_{3} \cdot w_{53}+O_{4} \cdot w_{54} \\
& \Rightarrow O_{5}=f\left(n e t_{5}\right)=\frac{1}{2} \cdot\left(1-n e t_{5}^{2}\right)
\end{aligned}
$$

İleri yönde hesaplama gerçekleştirildikten sonra elde edilen $O_{5}$ çıkış değeri $\Delta f$ miktarına karşılık gelen değerdir $\left(O_{5}=\Delta f\right)$. Eğitim setindeki $f_{t}$ ve $f_{h}$ giriş değerleri için beklenen çıkış değerini $d_{5}$ olarak adlandırırsak ağın bir iterasyondaki hata hesaplaması aşağıdaki gibi gerçekleştirilir.

$E=\frac{1}{2} \cdot\left(d_{5}-O_{5}\right)^{2}$

$E$ hata değeri beklenen hata değerinden büyük olduğu sürece eğitim iterasyonları aşağıdaki gibi tekrarlanır.

$$
\begin{aligned}
& \delta_{O_{5}}=\left(d_{5}-O_{5}\right) \cdot f^{\prime}(n e t)=\left(d_{5}-O_{5}\right) \cdot \frac{1}{2} \cdot\left(1-O_{5}^{2}\right) \\
& =\frac{1}{2} \cdot\left(d_{5}-O_{5}\right) \cdot\left(1-O_{5}^{2}\right) \\
& \delta_{O_{3}}=\left(\delta_{O_{5}} \cdot w_{53}\right) \cdot \frac{1}{2} \cdot\left(1-O_{3}^{2}\right) \\
& =\frac{1}{2} \cdot\left(\delta_{O_{5}} \cdot w_{53}\right) \cdot\left(1-O_{3}^{2}\right) \\
& \delta_{O_{4}}=\left(\delta_{O_{5}} \cdot w_{54}\right) \cdot \frac{1}{2} \cdot\left(1-O_{4}^{2}\right) \\
& =\frac{1}{2} \cdot\left(\delta_{O_{5}} \cdot w_{54}\right) \cdot\left(1-O_{4}^{2}\right)
\end{aligned}
$$

Bulunan bu değerler momentum katsayısı $(\eta)$ ve çıkış değerleri ile birlikte kullanılarak $w$ ağırlıkları aşağıdaki gibi güncellenir. Herhangi bir $t$ zamanda güncellenen ağırlıklar;

$$
\begin{aligned}
& w_{53}^{t}=w_{53}^{(t-1)}+\eta \cdot \delta_{O_{3}} \cdot O_{3} \\
& w_{54}^{t}=w_{54}^{(t-1)}+\eta \cdot \delta_{O_{4}} \cdot O_{4} \\
& w_{42}^{t}=w_{42}^{(t-1)}+\eta \cdot \delta_{O_{4}} \cdot O_{4} \\
& w_{30}^{t}=w_{30}^{(t-1)}+\eta \cdot \delta_{O_{3}} \cdot O_{3} \\
& w_{40}^{t}=w_{40}^{(t-1)}+\eta \cdot \delta_{O_{4}} \cdot O_{4} \\
& w_{31}^{t}=w_{31}^{(t-1)}+\eta \cdot \delta_{O_{3}} \cdot O_{3} \\
& w_{41}^{t}=w_{41}^{(t-1)}+\eta \cdot \delta_{O_{4}} \cdot O_{4} \\
& w_{32}^{t}=w_{32}^{(t-1)}+\eta \cdot \delta_{O_{3}} \cdot O_{3} \\
& w_{42}^{t}=w_{42}^{(t-1)}+\eta \cdot \delta_{O_{4}} \cdot O_{4}
\end{aligned}
$$



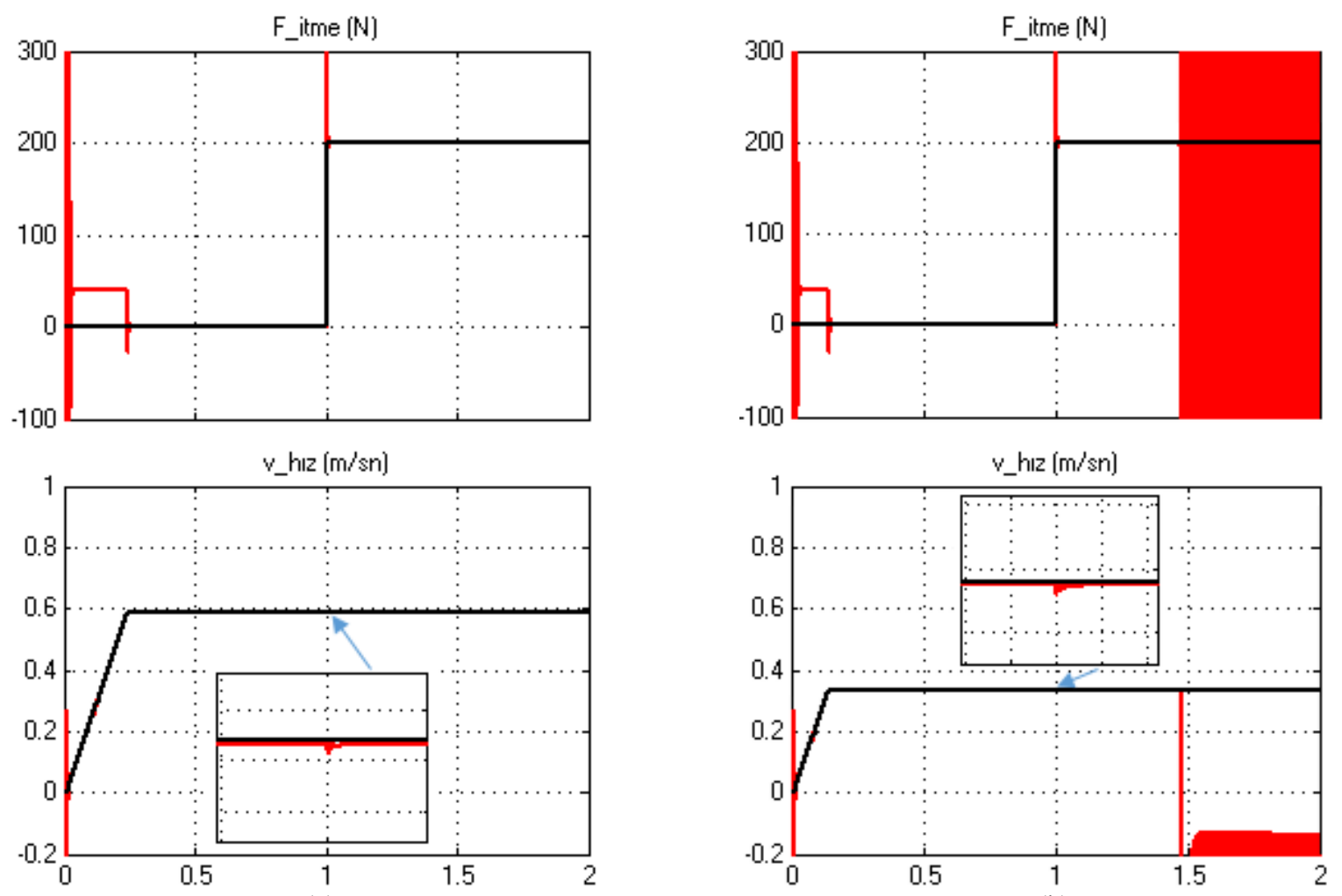

(a)

(b)

Şekil 8. Hız denetimi benzetim sonuçları (a) $40 \mathrm{~cm} / \mathrm{sn}$ üstü, (b) $40 \mathrm{~cm} / \mathrm{sn}$ altı (Speed control simulation results (a) 40 $\mathrm{cm} / \mathrm{sec}$ over, (b) $40 \mathrm{~cm} / \mathrm{sec}$ under)

Böylece ağırlıklar güncellenmiş olur. Beklenen $E$, hata değerine ulaşana kadar bu işlemler tekrarlanır.

Her deney öncesi YSA eğitimi gerçekleştirilir. Deneylerden elde edilen sonuçlar motor üzerinde bulunan Manyetik Lineer Enkoderden elde edilen (MLE) değerlerle karşılaştırılır. Motor üzerindeki MLE ve MLE verilerini gösteren Pic program devresi Şekil 6'da gösterilmiştir.

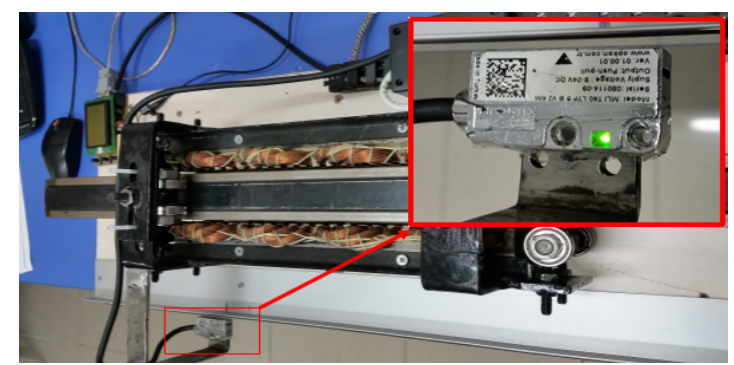

(a)

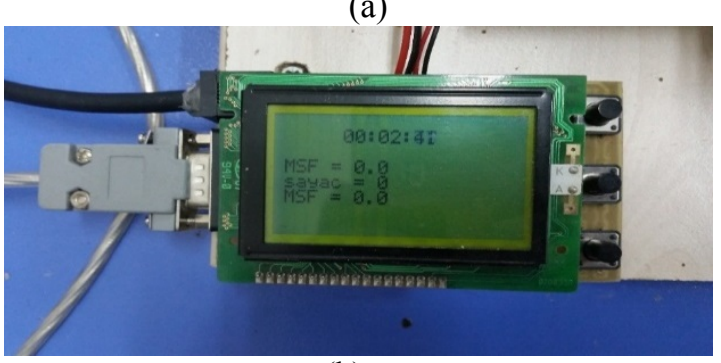

(b)

Şekil 6. Deneysel çalışmanın blok devre şeması (a) MLE, (b) Pic devre (The block diagram of the experimental studies (a) MLE (b) Pic circuit
Deneysel çalışmanın blok devre şeması Şekil 7'deki gibi gerçekleştirilmiştir.

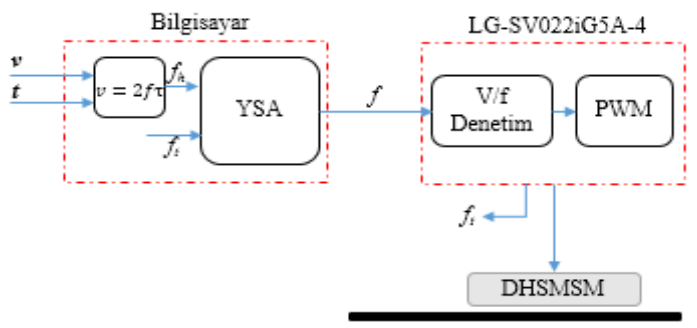

Şekil 7. Deneysel çalışmanın blok devre şeması (Block diagram of the experimental study)

\section{ELDE EDILEN SONUÇLAR VE DEĞERLENDIRME (OBTAINED RESULTS AND DISCUSSION)}

Deneysel çalışma öncesi DHSMSM'nin Matlab/Simulink programında bilgisayar benzetimi gerçekleştirilmiştir. Benzetimde kullanılan motor parametreleri ile deneyde kullanılan parametreler aynıdır. Benzetimde PID $+\mathrm{V} / \mathrm{f}$, deneyde $\mathrm{YSA}+\mathrm{V} / \mathrm{f}$ denetim yöntemleri kullanılmıştır. Böylece YSA tabanlı skaler denetimin düşük hızlardaki denetim başarımı incelenmiştir. Benzetim sonuçları Şekil 8'de verilmiştir.

Benzetim çalışmasında motor, verilen referans hızlara yüksüz ve yüklü durumlarda çok k1sa sürelerde ulaşmıştır. Ancak motor $40 \mathrm{~cm} / \mathrm{sn}$ hız değerinin altında iken başarılı sonuçlar üretmediği gözlenmiştir. 

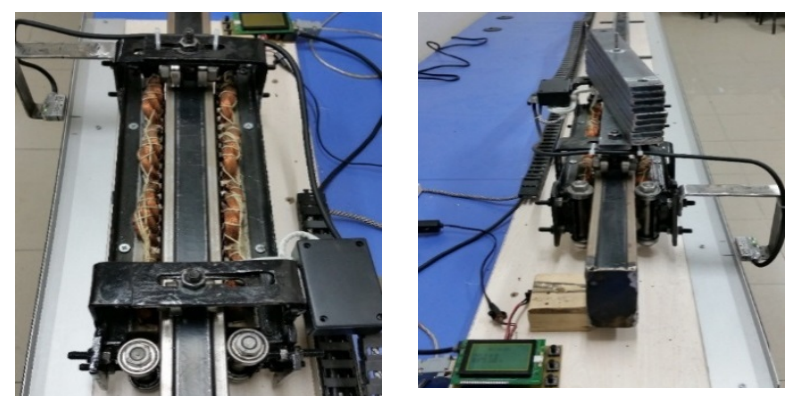

Şekil 9. Motorun yüksüz ve yüklü durumu (a) Motor yüksüz, (b) Motor yüklü (The unload and load condition of the motor (a) Motor unload, (b) Motor load)
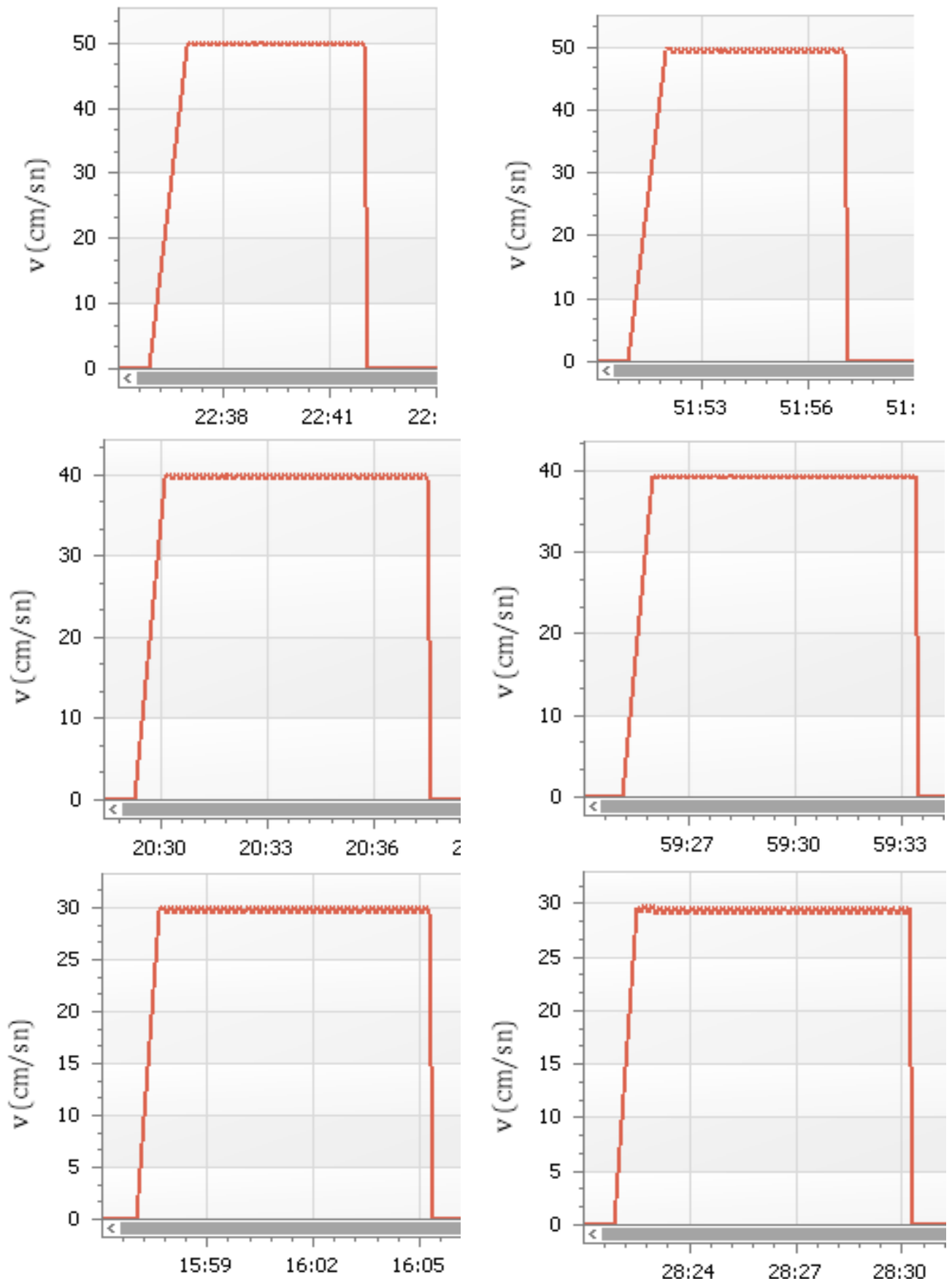

(a)

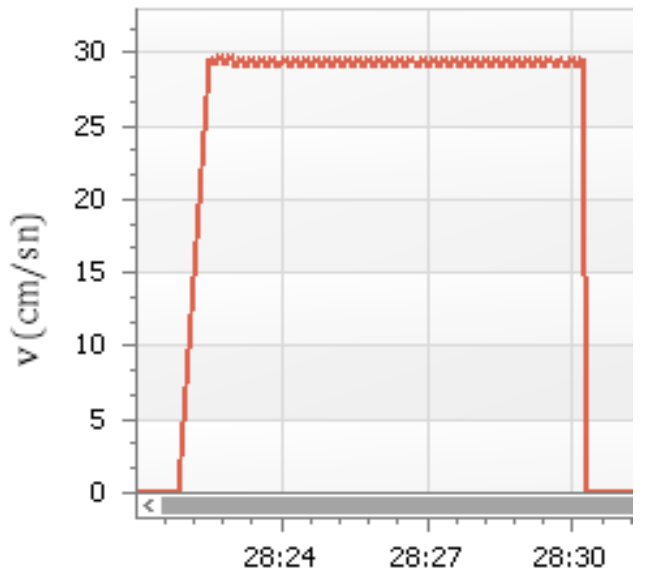

(b)

Şekil 10. Motorun yüksüz ve yüklü hız grafikleri (a) Motor yüksüz, (b) Motor yüklü (The unload and load speed graphics motor (a) Motor unload, (b) Motor load) 
Deneysel çalışmada, sürücü frekansı YSA ile denetlenmiştir. Motorun farklı hız ve yüklerdeki davranışları deney sonuçlarına göre değerlendirilmiştir. Motorun yüksüz ve yüklü durumu Şekil 9'de gösterilmiştir.

Şekil 9 (b)'de motora 10 kg harici yük konulmuştur. Şekil 10'da farklı hızlarda yüksüz ve yüklü durumlar için hız denetim grafikleri verilmiştir. Grafiklerin kolay analizi için yüklü ve yüksüz durum grafikleri yan yana verilmiştir.

Şekil 10 (a) sütununa ait grafikler motorun yüksüz durumda 50, 40 ve $30 \mathrm{~cm} / \mathrm{sn}$ hizlarda elde edilen grafiklerdir. Şekil 10 (b) sütununa ait grafikler ise motorun yüklü durumda 50,40 ve $30 \mathrm{~cm} / \mathrm{sn}$ hızlarda elde edilen grafikleri göstermektedir. Bu grafiklerde $40 \mathrm{~cm} / \mathrm{sn}$ hız değerinin altında motorun yüksüz ve yüklü gösterdiği hız denetim başarımı önemlidir. Bu durumda $30 \mathrm{~cm} / \mathrm{sn}^{\prime} l i k$ hiz grafikleri incelendiğinde; motor hızının yüksüz durumda $30.24 \mathrm{~cm} / \mathrm{sn}$ ve 29.48 $\mathrm{cm} / \mathrm{sn}$ aralığında olduğu ve istenen hıza ulaştığ görülmektedir. Motor yüklü durumda iken hız 29.82 $\mathrm{cm} / \mathrm{sn}$ ve $29.06 \mathrm{~cm} / \mathrm{sn}$ aralığında olduğu görülmektedir. $\mathrm{Bu}$ durumda motor yüksüz iken ortalama hızı $29.86 \mathrm{~cm} / \mathrm{sn}$, yüklü iken 29.44 $\mathrm{cm} / \mathrm{sn}$ 'dir. Böylece DHSMSM'nin istenen hıza yaklaşık \% 0.5 hata ile ulaştı̆̆ı anlaşılmaktadır.

\section{SONUÇLAR (CONCLUSIONS)}

Bu çalışmada DHSMSM'nin YSA tabanlı skaler hız denetimi önerilmiştir. Çalışmanın temelinde sürücü frekansı değiştirilerek motorun hı denetimi amaçlanmıştır. Geleneksel denetleyiciler düşük frekanslarda istenilen başarımı göstermediği için akıllı denetim sistemi çalışmada kullanılmıştır. Elde edilen deneysel sonuçlardan, motorun farklı hız ve yüklerde referans hızı yakaladığı ve düzgün bir şekilde bu hızı takip ettiği görülmüştür. Çalışmadan elde edilen sonuçlar aşağıdaki gibi özetlenebilir.

Motor sürücüsü endüstriyel sistemlerde kullanılan hazır bir sürücüdür. $\mathrm{Bu}$ sürücüye ait haberleşme protokolü dikkate alınarak bilgisayarda denetim programı ve arayüz hazırlanmıştır. $\mathrm{Bu}$ anlamda programda yapılabilecek küçük değişikliklerle denetim sistemimizin farklı bir sürücüye uyumu sağlanabilir.

Motorun yüksüz ve yüklü hı grafikleri arasında oluşan küçük farklılıkların motor yüklü durumda iken sürtünme kayıplarının artmasından kaynaklandığı söylenebilir. $\mathrm{Bu}$ durum sistem parametrelerine eklenerek giderilebilir. Ancak elde edilen sonuçlar bu hatanın ihmal edilebilir düzeyde olduğunu göstermektedir.

Deneylerde sürücü frekansı $1.5 \mathrm{~Hz}$ 'e ulaştığında motorun hareketi gözlenmiştir. Bu değerden düşük frekanslarda motorun hareket etmediği ve olduğu yerde titreşim gösterdiği görülmüştür. Buradan senkron frekansın yaklaşı $1.5 \mathrm{~Hz}$ olduğu anlaşılmaktadır. Başka bir ifadeyle, motordan elde edilen minimum hız (denklem 6 yardımıyla) yaklaşık $13 \mathrm{~cm} / \mathrm{sn}$ 'dir.

Önerilen yöntemin, son y1llarda teknolojinin gelişimiyle birlikte kullanımı artan DHSMSM'lerin hız denetiminde kullanılabileceğini göstermektedir.

Yapılan çalışmalar halen birçok ülkede aktif araştırma konusu olduğundan bu çalışmanın da literatüre katkıda bulunacağı düşünülmektedir.

\section{KAYNAKLAR (REFERENCES)}

1. Irmak, E. ve Vadi, S., "Asenkron Motorlarda Frekans Değişimi ile $\mathrm{H} ı$ Kontrolü Deneyinin Bilgisayar Üzerinden Gerçekleştirilmesi”, Journal of The Faculty of Engineering and Architecture of Gazi University, Cilt 21, No 1, 57-62, 2011.

2. Gieras, J. F. ve Piech, Z. J., Linear Synchronous Motors Transportation and Automation Systems, CRC Press, Boca Raton, Florida, 2000.

3. Boldea, I., Linear Electric Machines, Drives, and MAGLEVs Handbook, CRC Press, Boca Raton, London, 2013.

4. Karaçar, E., "Hidrolik ve Pnömatik'e Alternatif Çözüm: Doğrusal Motorlar”, II. Ulusal Hidrolik ve Pnömatik Kongresi ve Sergisi, İzmir, 2001.

5. Cheema, M. ve Fletcher, J. E., "Sensorless Vector Control of Linear Permanent Magnet Synchronous Motor", ECCE Asia Downunder (ECCE Asia), Melbourne, Avustralya, 1094-1108, 3-6 Haziran 2013.

6. Ponomarev, P., Control of Permanent Magnet Linear Synchronous Motor in Motion Control Applications, Master Tezi, Lappeenranta University of Technology, Faculty of Technology, Lappeenranta: Department of Electrical Engineering, 2009.

7. Balazovic, P., 3-Phase PM Synchronous Motor Torque Vector Control, Czech Republic: Motorola, DRM018, Motorola Czech System Laboratories Roznov pod Radhostem, 2003.

8. Boldea, I. ve Nasar, S. A., Linear Electric Actuators and Generators, Cambridge University Press, London, (U.K.), 1997.

9. Demir, K., Doğrusal Hareketli Asenkron Motor Tasarımı ve Uygulaması, Seminer Notları, Trabzon, Karadeniz Teknik Üniversitesi,Fen Bilimleri Enstitüsü, Mayis 2012.

10. Altun, H., "Matris Çeviriciden Beslenen Uç Etkili Lineer Senkron Motorun Kontrol Simülasyonu", Journal of The Faculty of Engineering and Architecture of Gazi University, Cilt 26, No 2, 359372, 2011.

11. Röhrig, C., Jochheim, A., "Motion Control of Linear Synchronous Motors With Force Ripple Compensation Using Current Shaping", 15th Triennial World Congress, Barcelona, Spain, 1480, 2002.

12. Küçükali, M., Doğrusal Hareketli Asenkron Motor Sürücülü Mekanik Osilatör ve Evirici ile Kontrolü, 
Master Tezi, Trabzon, Karadeniz Teknik Üniversitesi, Fen Bilimleri Enstitüsü, Temmuz 2008.

13. Fernandes, T. R., Short Primary Linear Drive Designed for Synchronous and Induction Operation Mode with On-Board Energy Storage, Technischen Universitat, Darmstadt, 2012

14. Bizot, C., Brottes, J., Lungeanu, M., Poulsen, B., Séra, D. ve Sørensen, M. B., "Sensorless Control for PMSM", Power Electronics and Drives, Aalborg University, Institute of Energy Technology, Denmark, 2003

15. Yıldız, C., Genetik Algoritma Destekli Bulanık Denetim Kullanarak Vektör Esaslı Asenkron Motor Kontrolü, Master Tezi, Kahramanmaraş Sütçü İmam Üniversitesi, Fen Bilimleri Enstitüsü, Kahramanmaraş, 2008.

16. Haitham, A., Atif, I. ve Jaroslaw, G., High Performance Control of AC Drives With Matlab/Simulink Models, Wiley, Noida, India, 2012.

17. Sala-Perez, P. ve Galceran-Arellano, S., "A sensorless stable V/f control method for a five-phase PMSM", Power Electronics and Applications (EPE), 15th European Conference on, 1-10, 2-6 Eylül 2013.

18. Limci, Z., Chan H., Ham, Q., Han, Thomas X., Wu, L., Zheng, Sundaram, K. B., Kapat, J. and How, L., "Design of an optimal V/f control for a super high speed permanent magnet synchronous motor", The 30th annual conferance of the IEEE Industrial Electronic, Busen, Korea, 2260 - 2263, 2-6 Kasim 2004.

19. $\mathrm{Lu}, \mathrm{H} ., \mathrm{Li}, \mathrm{M}$. ve $\mathrm{Xu}, \mathrm{Y} .$, "Sensorless position and speed estimation with improved integrator for PMLSM drive", Information and Automation, ICIA '09. International Conference on, Ohio, 380 - 384, 22-24 Haziran 2009.

20. Garcia, G. O., Stephan, R. M. ve Watanabe, E. H., "Comparing the indirect field-oriented control with a scalar method", IEEE Trans. Ind. Appl., Cilt 2, No 41, 201-207, 1994.

21. Itoh, J.-I., Nomura, N. ve Ohsawa, H., "A comparison between $\mathrm{V} / \mathrm{f}$ control and position-sensorless vector control for the permanent magnet synchronous motor", Power Conversion Conference, PCC-Osaka, 13101315, 2002.

22. Jafari, S., Corzine, K. ve Huang, J., "Efficiency optimization of a sensorless V/f control method for PMSM", Sensorless Control for Electrical Drives (SLED), 2012 IEEE Symposium on, Milwaukee, WI., 1-5, 21-22 Eylül 2012.

23. Kavuran, G. Orhan, A., "Open-loop speed Control of Permanent Magnet Synchronous Motor Fed by Matrix Converter", Electrical, Electronics and Computer Engineering (ELECO), Bursa, 334 - 338, 2-5 Aralık 2010.

24. Zhao, L., Ham, C.H., Han, Q., Wu, Thomas X., Zheng, L., Sundaram, K.B. Kapat, J., Chow, L., "Design of an optimal V/f control for a super high speed permanent magnet synchronous motor", Industrial Electronics Society, IECON 2004. 30th Annual Conference of IEEE, Busan, Korea, 2260 - 2263, 2-6 Kasım 2004.

25. Junyou, Y., Jiefan, C., Guofeng, H., "Direct Thrust Control of PMLSM Using SVM and Sliding Mode Variable Structure", J. Transactions of China Electrotechnical Society, Cilt 22, No 6, 24-29, 2007.
26. Gireas, J. F., Piech, Z. J. ve Tomzcuk, B. Z., Linear Synchronous Motors: Transportation and Automation Systems, CRC Press, Florida, 2013.

27. Zhang, Y., Song, J., Song, S., Yan, M., “Adaptive PID Speed Controller Based on RBF for Permanent Magnet Synchronous Motor System", International Conference on Intelligent Computation Technology and Automation, Changsha, China, 2260-2263, 2-6 Kasim 2010.

28. Huacai, L., Yuetong, X., Weimin, Y., Zichen, C., "Fuzzy PID Controller Design for a Permanent Magnet Linear Synchronous Motor Feeding System", J. Transactions of China Electrotechnical Society, Cilt 22, No 4, 59-63, 2007.

29. Srinivasu, B., Prasad, P. ve Ramana Rao, M., "Adaptive Controller Design for Permanent Magnet Linear Synchronous Motor Control System", New Power Electronics, Drives and Energy Systems, PEDES '06, International Conference on, Delhi, India, 1-6, 12-15 Aralık 2006.

30. Zare, J., "Vector Control of Permanent Magnet Synchronous Motor With Surface Magnet Using Artificial Neural Networks", 43rd International Universities Power Engineering Conference, UPEC, Padova, Italy, 1-4, 1-4 Eylül 2008.

31. Kumar, R., Gupta, R. A. ve Bansal, A. K., "Identification and Control of PMSM Using Artificial Neural Network", IEEE International Symposium on Industrial Electronics (ISIE), Vigo, Spain, 30-35, 4-7 Haziran 2007.

32. Panah, P. G., Shafiei, A. ve Sharifian, R., "An improved variable structure design for velocity control of a permanent magnet linear synchronous motor", Power Electronics Electrical Drives Automation and Motion (SPEEDAM), 2010 International Symposium on, Naples, Italy, 1231-1236, 14-16 Haziran 2010.

33. Verma, A., Singh, B. ve Yadav, D., "Investigation of ANN tuned PI speed controller of a modified DTC induction motor drive", Power Electronics, Drives and Energy Systems (PEDES), 2014 IEEE International Conference on, 1-6, 2014.

34. Așkın, D., İskender, İ. ve Mamızadeh, A., "Farklı Yapay Sinir Ağları Yöntemleri Kullanarak Kuru Tip Transformatör Sargısının Termal Analizi”, Journal of The Faculty of Engineering and Architecture of Gazi University, Cilt 26, No 4, 905-913, 2011.

35. Wei, W., YuHua, W., ShiRong, W., "A Speed Control System of Permanent Magnet Linear Synchronous Motor Using Neuron Adaptive Controller", Second International Conference on Intelligent Computation Technology and Automation, Washington, DC, USA, 35-38, 10-11 Ocak 2009.

36. Hamidia, F., Boucherit, M., Larabi, A. ve Bouhedda, M., "Direct torque control with ANN speed controller based on Kalman filter for PMSM", Systems, Signals and Devices (SSD), 9th International MultiConference on , Chemnitz, Germany, 2012.

37. Elmas, Ç., Yapay Sinir Ağları, Seçkin yayıncılık, Ankara, 2003.

38. Chaoui, H., Gueaieb, W. ve Yagoub, M., "Neural network based speed observer for interior permanent magnet synchronous motor drives", Electrical Power \& Energy Conference (EPEC), Canada, 1-6, 22-23 Ocak 2009. 
39. Otkun, Ö. ve Akpınar, A. S., "Çift Yanlı Doğrusal Hareketli Sürekli Miknatıslı Senkron Motorun Parametrelerinin Belirlenmesi”, Pamukkale University Journal of Engineering Sciences, Cilt 21, No 2, 52-58, 2015.

40. Jacek, N. Z., Introduction To Artificial Neural Systems, West Publishing Company, St. Paul, 1992
41. Gaur, P., Singh, B. ve Mittal, A., "Artificial Neural Network based Controller and Speed Estimation of Permanent Magnet Synchronous Motor", Power System Technology and IEEE Power India Conference, POWERCON 2008. Joint International Conference on, New Delhi, India, 1-6, 2008. 